\title{
ANÁLISE ESTATÍSTICA DA NÃO ESTACIONARIEDADE DE SÉRIES TEMPORAIS DE VAZÃO MÁXIMA ANUAL DIÁRIA NA BACIA HIDROGRÁFICA DO RIO PARDO
}

\author{
C. A. SANTOS ${ }^{1 *}$, A. M. M. LIMA ${ }^{2}$, M. H. C. S. FARIAS ${ }^{3}$, U. R. V. AIRES ${ }^{1}$, E. A. O. SERRÃO 4 \\ ${ }^{1}$ Universidade Federal de Viçosa (UFV) \\ ${ }^{2}$ Universidade Federal do Pará (UFPA) \\ ${ }^{3}$ Universidade do Estado do Pará (UEPA) \\ ${ }^{4}$ Universidade Federal de Campina Grande (UFCG) \\ cleber_ufpa@yahoo.com.br
}

Artigo submetido em julho/2016 e aceito em setembro/2016

DOI: $10.15628 /$ holos.2016.4892

\section{RESUMO}

Nos últimos anos, a variabilidade climática e o uso desordenado do solo têm impactado vários componentes do ciclo hidrológico em muitas regiões em todo o mundo, fazendo com que as séries hidrológicas deixem de ser estacionárias. Este trabalho tem por finalidade identificar se as vazões máximas anuais diárias da bacia hidrográfica do Rio Pardo apresentam comportamento não estacionário e o principal motivo de tal fator. Os dados pluviométricos e fluviométricos de vazão foram obtidos a partir do banco de dados históricos disponibilizados pela Agência Nacional de Águas (ANA), entre os anos de 1941 a 2013. Os dados de uso do solo, para caracterização da área antropizada são provenientes do Grupo de Pesquisa em Interação Atmosfera-Biosfera da UFV, onde foram utilizados apenas os dados de pastagens plantadas como classificação do uso do solo de 1940 a 2010. Os testes estatísticos empregados para a identificação da não estacionariedade foram os testes não paramétricos de Mann-Kendall e Spearman Rho, além do teste de Pettitt para localizar o ponto de ruptura na série. Foi identificada uma tendência crescente na série de vazão anual máxima diária do Rio Pardo, logo, esta não se caracterizou como estacionária. Como os eventos de precipitação estão estatisticamente estacionários, a substituição da vegetação natural por pastagens plantadas, representa um dos principais fatores do comportamento da série de vazão máxima não ser mais estacionária.

PALAVRAS-CHAVE: Hidrologia, Uso do solo, Teste de tendência, Ponto de mudança.

\section{STATISTICAL ANALYSIS OF NON-STATIONARY TIME SERIES OF ANNUAL MAXIMUM DAILY FLOW IN THE PARDO RIVER BASIN}

\section{ABSTRACT}

In recent years, climate variability and disorderly land use have impacted in various components of the hydrological cycle in many regions around the world, making the hydrologic series stop to be stationary. This study aims to identify if the annual maximum daily flow in the Pardo River Basin showing non-stationary behavior and the main reason for this factor. The rainfall and streamflow data of flow were obtained from the historical database provided by the National Water Agency (ANA), between the years 1941-2013. The land use data utilized to characterize the anthropic area are from the Research Group in Atmosphere-Biosphere Interaction of Federal University of Viçosa, which were
\end{abstract}

used only the pastures planted data as classification of land use from 1940 until 2010. The statistical tests utilized for identification of non-stationarity were the non-parametric tests of Mann-Kendall and Spearman's Rho, besides Pettitt test to locate the break point in the serie. It was identified an increasing trend in the series of the annual maximum daily flow in the Pardo River Basin, so it was not characterized as stationary. As precipitation events are statistically stationary, the replacement of natural vegetation by planted pastures represents one of the main factors of the maximum flow serie not be considered as stationary.

KEYWORDS: Hydrology, Land use, Trend test, Turning point. 


\section{INTRODUÇÃO}

Nos últimos anos, a variabilidade climática e o uso desordenado do solo têm impactado vários componentes do ciclo hidrológico em muitas regiões em todo o mundo, fazendo com que as séries hidrológicas deixem de ser estacionárias (MILLY et al., 2008). De acordo com Tucci (2002), uma série é dita estacionária quando suas estatísticas não se alteram com o tempo e as amostras utilizadas são representativas, logo não há tendência nem de redução nem de aumento.

Por sua importância econômica e ambiental, a vazão é uma das variáveis hidrológicas mais estudadas. Vários usos da água são altamente dependentes dos padrões de vazão, servindo de fonte mais fácil e mais acessível na sua utilização para consumo humano, animal, irrigação e outros usos (VILANOVA, 2014).

A não estacionariedade em séries hidroclimatológicas podem estar ligadas a diversos fatores. Estudos apontam (ADNAN e ATKINSON 2010; KOCHNDORFER e HUBBART, 2010; HE et al., 2013; VILANOVA, 2014; DALE et al., 2015; KIBRIA et al., 2016) que as tendências em séries históricas de vazão estão intimamente ligadas com a atuação conjunta (ou separada) do uso do solo e alterações no regime pluviométrico.

Diversos autores têm investigado a existência da não estacionariedade em séries de vazões máximas (WANG et al., 2005; SALVADORI, 2013; DAMÁZIO e COSTA 2014; TAN e GAN, 2015). Entretanto, padrões de mudanças de vazão regionais são complexos (MILLY et al., 2005), o que pode levar a incertezas de qual fator está contribuindo significativamente nas alterações. Alguns estudos como o de Labat et al. (2004) e Gedney et al. (2006), mostram a relação do aumento da vazão com as mudanças no clima, através da influência direta da precipitação, outros autores atribuem a ligação do aumento da vazão com alterações na cobertura vegetal (BOSCH e HEWLETT, 1982).

Uma possível região onde pode estar ocorrendo a não estacionariedade em séries hidrológicas é o centro Sul do Brasil, em especial a bacia hidrográfica do Rio Pardo, localizado entre os Estados de São Paulo e Minas Gerais. Por ser uma região onde o desenvolvimento agrícola é bastante acentuado, possivelmente o uso irregular do solo possa provocar alterações no ciclo hidrológico da bacia, já que de acordo com Celarino (2015), esta região, além de outras culturas agrícolas, apresenta agricultura focada na produção da cana-de-açúcar (Saccharum officinarum).

A evidência da não estacionariedade de alguns registros hidrológicos longos têm levantado uma série de questões quanto à adequação dos métodos estatísticos convencionais para a previsão de recursos hídricos regionais de longo prazo (COULIBALY e BALDWIN, 2005).

Análises estatísticas de tendência podem ser utilizadas para identificar a não estacionariedade em séries de dados de vazão, como o teste de Mann-Kendall e Spearman rho (KAHYA e KALAYCI, 2004; DIERMANSE et al., 2010; DETZEL e MINE, 2014) e onde ocorreu o ponto de mudança na série, utilizando o teste de Pettitt (SALVADORI, 2013; YEH et al., 2015).

Diante do exposto, o objetivo do trabalho é identificar se as vazões máximas anuais diárias da bacia hidrográfica do Rio Pardo apresentam comportamento não estacionário e o principal motivo de tal fator. 


\section{MATERIAIS E MÉTODOS}

\section{1 Área de estudo}

A bacia hidrográfica do Rio Pardo está localizada entre os Estados do São Paulo e Minas Gerais, drenando uma área de aproximadamente $12.728,29 \mathrm{~km}^{2}$. A nascente do rio principal localiza-se no município de Ipuiuna - MG e seu comprimento total, gira em torno de $378,82 \mathrm{~km}$. A estação fluviométrica está localizada no município de Ribeirão Preto - SP (Clube de Regatas, código 61834000) (Figura 01).

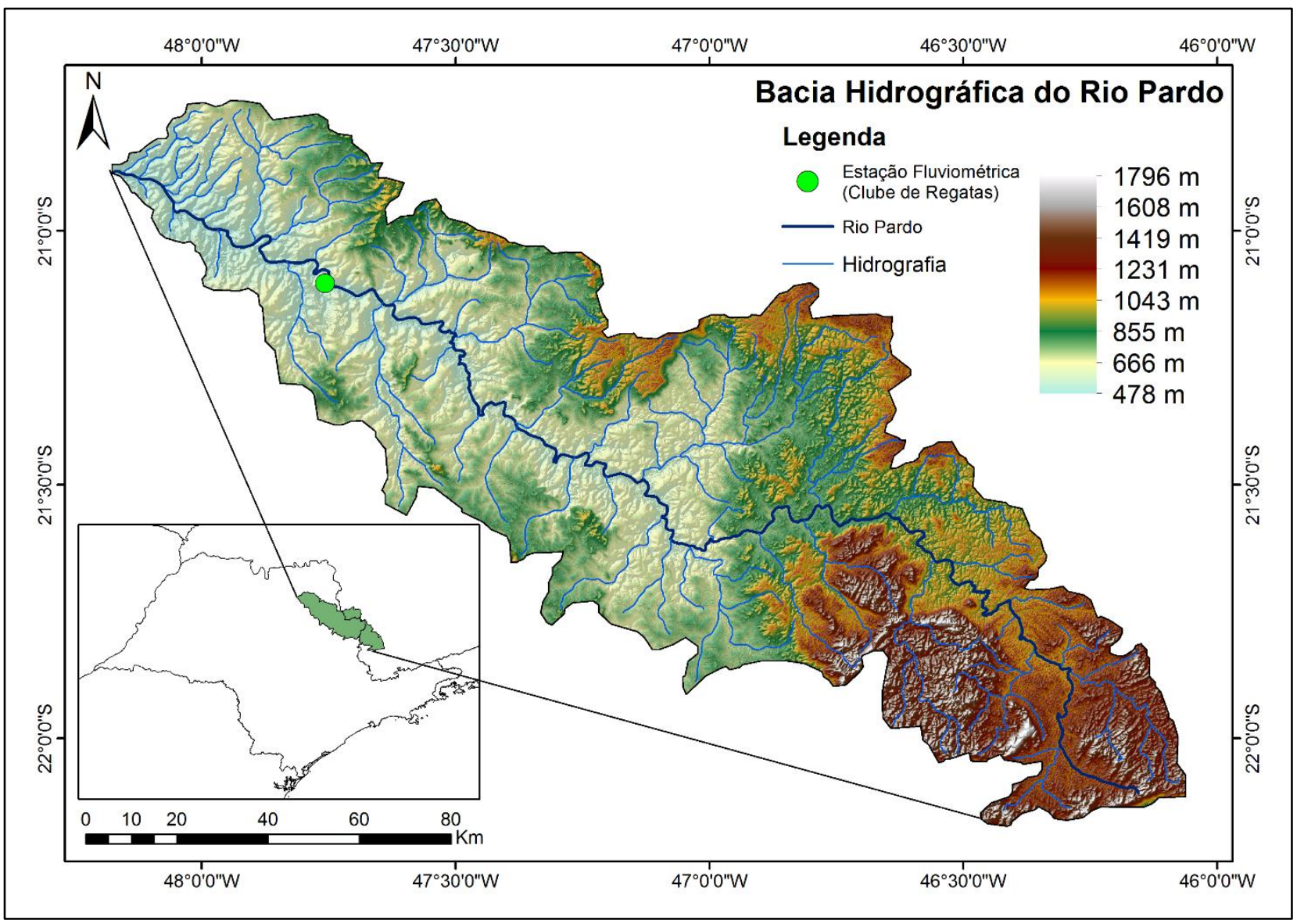

Figura 1: Localização, topografia, hidrografia e estação fluviométrica da bacia hidrográfica do Rio Pardo. Fonte: Base vetorial da ANA/IBGE (2010) e modelo digital de elevação do SRTM.

A região do alto curso da bacia, apresenta as maiores elevações topográficas, alcançando o máximo de $1796 \mathrm{~m}$, caracterizado por vales bastante entalhados com pouca dimensão interfluvial, sendo comum a presença de corredeiras (CELARINO, 2015). No médio e baixo curso, ocorre a diminuição da declividade e da velocidade da água nos canais, onde a bacia se torna relativamente plana, em comparação ao alto curso, podendo alcançar o valor mínimo de elevação topográfica de $478 \mathrm{~m}$.

De acordo com as normais climatológicas (1961-1990) do Instituto Nacional de Meteorologia (INMET), a precipitação média anual acumulada na bacia do Rio Pardo é de 1550 $\mathrm{mm} / \mathrm{ano}$, onde o mês que concentra maior volume precipitado é dezembro ( $280 \mathrm{~mm}$ ), principalmente por sofrer a atuação da Zona de Convergência do Atlântico Sul (ZCAS) nesse 
período (GAN et al., 2004) e o mês com o menor volume de precipitação na bacia é agosto ( 30 $\mathrm{mm})$.

\subsection{Dados utilizados}

\subsubsection{Dados fluviométricos}

Os dados fluviométricos de vazão $\left(\mathrm{m}^{3} / \mathrm{s}\right)$ foram obtidos a partir do banco de dados históricos disponibilizados pela Agência Nacional de Águas (ANA), por meio da estação do Clube de Regatas (código 61834000) (Figura 1). Esta estação conta com dados de 01/1941 a 12/2013. Através do software Hidro 1.2 da ANA, foram extraídos os valores máximos diários de vazão para cada ano, totalizando 73 valores.

\subsubsection{Dados pluviométricos}

Os dados pluviométricos $(\mathrm{mm})$ são provenientes do banco de dados históricos da ANA. Foram utilizadas as médias de 10 estação pluviométricas dentro e no entorno da bacia hidrográfica do Rio Pardo sendo 6 estações com período de 1941 a 2013 e 4 estações com período entre 1941 a 2000 (Tabela 1). Utilizou-se apenas os acumulados mensais pertencentes ao período do verão austral (dezembro, janeiro e fevereiro) por apresentarem maior significância nos eventos de vazão máxima.

Tabela 1: Dados das estações pluviométricas da bacia hidrográfica do Rio Pardo.

\begin{tabular}{|c|c|c|c|c|}
\hline Estação & Código & Latitude & Longitude & Período \\
\hline São Sebastião da Grama & 02146010 & $-21^{\circ} 42^{\prime} 00^{\prime \prime}$ & $-46^{\circ} 49^{\prime} 00^{\prime \prime}$ & $1941-2013$ \\
\hline Santa Cruz das Palmeiras & 02147033 & $-21^{\circ} 50^{\prime} 00^{\prime \prime}$ & $-47^{\circ} 15^{\prime} 00^{\prime \prime}$ & $1941-2000$ \\
\hline Ribeirão Preto & 02147006 & $-21^{\circ} 13^{\prime} 00^{\prime \prime}$ & $-47^{\circ} 52^{\prime} 00^{\prime \prime}$ & $1941-2013$ \\
\hline Pontal & 02148003 & $-21^{\circ} 01^{\prime} 00^{\prime \prime}$ & $-48^{\circ} 02^{\prime} 00^{\prime}$ & $1941-2000$ \\
\hline Orlândia & 02047025 & $-20^{\circ} 44^{\prime} 00^{\prime \prime}$ & $-47^{\circ} 53^{\prime} 00^{\prime \prime}$ & $1941-2013$ \\
\hline Morro Agudo & 02048023 & $-20^{\circ} 44^{\prime} 00^{\prime \prime}$ & $-48^{\circ} 03^{\prime} 00^{\prime \prime}$ & $1941-2013$ \\
\hline Mococa & 02147023 & $-21^{\circ} 27^{\prime} 00^{\prime \prime}$ & $-47^{\circ} 00^{\prime} 00^{\prime \prime}$ & $1941-2000$ \\
\hline Fazenda Conquista & 02047027 & $-20^{\circ} 48^{\prime} 00^{\prime \prime}$ & $-47^{\circ} 46^{\prime} 00^{\prime \prime}$ & $1941-2013$ \\
\hline Fazenda Açude & 02146003 & $-21^{\circ} 26^{\prime} 00^{\prime \prime}$ & $-46^{\circ} 51^{\prime} 00^{\prime \prime}$ & $1941-2000$ \\
\hline Cravinhos & 02147014 & $-21^{\circ} 22^{\prime} 00^{\prime \prime}$ & $-47^{\circ} 43^{\prime} 00^{\prime \prime}$ & $1941-2013$ \\
\hline
\end{tabular}

\subsubsection{Dados de uso do solo}

Os dados de uso do solo, foram elaborados por Dias et al. (2016), referente ao período de 1940 a 2012. Estes dados representam as terras de cultivos e pastagens no Brasil, através da reconstrução histórica dos dados combinados dos censos agrícolas do Instituto Brasileiro de Geografia e Estatística (IBGE) e dados de sensoriamento remoto do Landsat. Os mapas de pastagem são divididos em pastagens plantadas e naturais (1940 a 2012), e mapas de terras de cultivo são divididos nas três principais culturas cultivadas no Brasil, a cana-de-açúcar, a soja (Glycine max) e o milho (Zea mays) (1990-2012). Neste trabalho, foram utilizados apenas os dados de pastagens plantadas como classificação do uso do solo.

A resolução espacial dos dados é de 30" ( 1 km). Estes dados foram baixados (http://www.biosfera.dea.ufv.br/) no formato ".nc" e processados no software ArcMap 10.0 para 
a classificação da porcentagem das áreas antropizadas na bacia e elaboração dos mapas de uso do solo.

\subsection{Estatística empregada}

\subsubsection{Teste de tendência}

Existem vários métodos estatísticos disponíveis para testar a estacionaridade de séries temporais em hidrologia, dentre eles, destacam-se os testes não paramétricos de Mann-Kendall (MANN, 1945; KENDALL, 1975) e Spearman Rho (LEHMANN, 1975; SNEYERS, 1990). De acordo com Wang et al. (2005), o teste de tendência para séries anuais nos dá uma visão global da mudança nos processos de vazão.

O teste de Mann-Kendall consiste em um teste estatístico não-paramétrico aplicado na identificação de tendências significativas de longo prazo, é comumente utilizado em análises de séries hidrometeorológicas, demonstrando bastante robustez quanto aos desvios de normalidade e não-estacionalidade dos valores de uma série (YUE et al., 2002; SANCHES et al., 2013; AHMAD et al., 2015).

Para um conjunto de dados, $X_{1}, X_{2}, X_{3}, \ldots, X_{n}$, com $n>4$, em que $n$ é o número de pontos de dados para a comprovação da hipótese nula HO (onde não há tendência positiva nem negativa na série) ou de hipótese alternativa $\mathrm{H}_{1}$ (onde há tendência positiva ou negativa na série). As equações matemáticas para o cálculo das estatísticas do teste de Mann-Kendall $S$ (Equação 1 ), $V(S)$ (Equação 2) e padronização a estatística do teste $Z$ (Equação 3) são os seguintes:

Através da consideração da hipótese nula $\left(\mathrm{H}_{0}\right)$, temos o somatório de $x_{j}$ e $x_{k}$ que são as séries de observações temporais em ordem cronológica, $n$ é o comprimento da série temporal:

$$
S=\sum_{k=1}^{n-1} \sum_{j=k+1}^{n} \operatorname{sign}\left(x_{j}-x_{k}\right)
$$

onde,

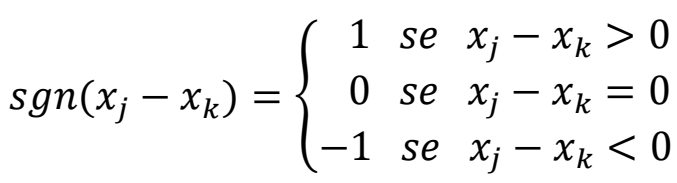

Partindo da premissa de que a hipótese $\mathrm{H}_{0}$ seja verdadeira, é possível mostrar que $\mathrm{S}$ é normalmente distribuída usando a variância de S:

$V(S)=\frac{1}{18}\left[n(n-1)(2 n+5)-\sum_{p=1}^{g} t_{p}\left(t_{p}-1\right)\left(2 t_{p}+5\right)\right]$ 
Onde $\mathrm{n}$ e número de observações, g é o número de grupos vinculados (um grupo amarrado é um conjunto de dados de amostra com o mesmo valor) e $t_{p}$ é o número de é o número de observações iguais no grupo $p$.

Logo a estatística Z do teste de Mann-Kendall foi então calculada:

$Z(M K)=\left\{\begin{array}{ccc}\frac{S-1}{\sqrt{V A R(S)}} & \text { se } & S>0 \\ 0 & \text { se } & S=0 \\ \frac{S+1}{\sqrt{V A R(S)}} & \text { se } & S<0\end{array}\right.$

Onde o valor positivo de $Z$ indica uma tendência ascendente, enquanto um $Z$ negativo indica uma tendência descendente. O nível de significância $\alpha$ adotado é de $\alpha=0,05=5 \%$ (para valores menores que 1,96, aceita-se a hipótese nula) para o teste de Mann-Kendall. Se a probabilidade $p$ do teste de Mann-Kendall for menor que o nível $\alpha, p<\alpha$, uma tendência estatisticamente significante existe, enquanto, um valor de $p>\alpha$, confirma uma tendência insignificante.

$O$ teste de Spearman rho é outro método não-paramétrico baseado em classificação utilizado para análise de tendências (YUE et al., 2002; SHADMANI et al., 2012; QUEIROZ, 2013; AHMAD et al., 2015; ZHANG et al., 2015). Neste teste, assume-se que os dados de séries temporais são independentes e identicamente distribuídos, a hipótese nula $\left(\mathrm{H}_{0}\right)$ novamente indica nenhuma tendência ao longo do tempo e a hipótese alternativa $\left(\mathrm{H}_{1}\right)$ é que uma tendência existe crescentes ou decrescente na série. No teste estatístico $D_{s r}$ é a estatística padronizada de $Z_{s r}$, são definidas como (Equações 4 e 5):

$D_{s r}=1-\frac{6 \sum_{i=1}^{n}\left(R_{i}-i\right)^{2}}{n\left(n^{2}-1\right)}$

$Z_{s r}=D_{s r} \sqrt{\frac{n-2}{1-D_{s r}^{2}}}$

Onde $R_{i}$ é a classificação das observações de $i ; n$ é o tamanho da série histórica. O nível de significância adotado foi o mesmo proposto no teste de Mann-Kendall.

\subsubsection{Ponto de mudança}

Há uma variedade métodos estatísticos para a detecção de um ponto de mudança em uma série temporal (SHARKEY e KILLICK, 2014; VEZZOLI et al., 2012). Neste estudo, será utilizado o teste 
não paramétrico proposto por Pettitt (1979) para detectar a ocorrência da mudança abrupta. 0 nível de significância adotado foi de $5 \%$.

O teste de Pettitt utiliza uma versão do teste de Mann-Whitney $U_{t, T}$, o qual verifica se duas amostras $X_{1}, \ldots, X_{t}$ e $X_{t}+1, \ldots, X_{n}$, são da mesma população ou não (PENEREIRO e FERREIRA 2012; ULIANA et al., 2015). A estatística de teste conta o número de vezes que o valor da primeira amostra excede o valor da segunda amostra. A hipótese nula $\left(\mathrm{H}_{0}\right)$ do teste de Pettitt admite ausência de um ponto de mudança na série (ULIANA et al., 2015), contra a alternativa $\left(\mathrm{H}_{1}\right)$ de existe um ponto de mudança. A estatística não paramétrica é definida como (Equações 6 e 7):

$$
K_{T}=\max _{1 \leq t \leq T}\left|U_{t, T}\right|
$$

onde,

$$
U_{t, T}=\sum_{i=1}^{t} \sum_{j=t+1}^{T} \operatorname{sgn}\left(X_{i}-X_{j}\right)
$$

em que:

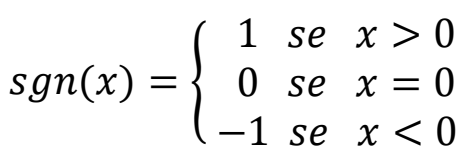

Logo, a estatística $U_{t, T}$ é calculada para valores de $1 \leq \mathrm{t} \leq \mathrm{T}$, e a estatística $K_{T}$ do teste é o máximo valor absoluto de $U_{t, T}$. Essa estatística localiza o ponto em que houve uma mudança brusca na média de uma série temporal (PENEREIRO e FERREIRA 2012), onde sua significância é obtida através da Equação (8):

$$
P \cong 2 \exp \left(\frac{-6 K_{T}^{2}}{T^{3}+T^{2}}\right)
$$

Onde $\mathrm{P}$ é o nível de significância; $K_{T}$ é o valor crítico; $T$ é o número de anos da série histórica. Quando $P$ é menor do que o nível de significância específica neste estudo $(0,05)$, a hipótese nula é rejeitada. 


\section{MODELO DE FORMA DE APRESENTAÇÃO DO ARTIGO}

\subsection{Identificação de não estacionariedade para a vazão máxima anual}

\subsubsection{Tendências na série de vazão máxima anual}

As vazões máximas anuais durante o período analisado (1941-2013) estão apresentadas na Figura 2. Podemos observar um aumento gradativo na série histórica.

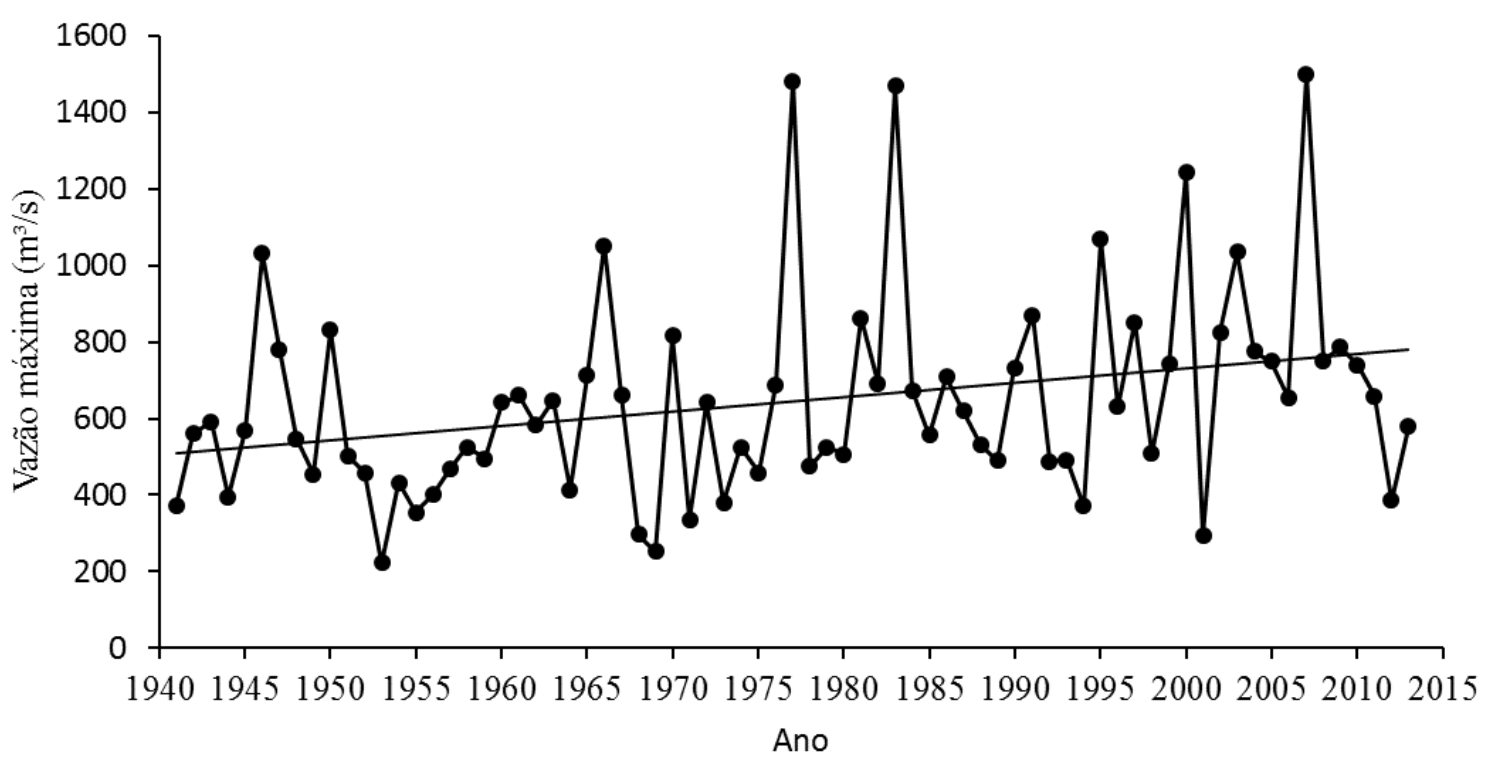

Figura 2: Vazão máxima anual do Rio Pardo na estação Clube de Regatas. Fonte: Adaptado da ANA (2016).

De acordo com Bayern (2014) esse tipo de comportamento informa que a série pode não ser estacionária, devido alterações no nível da série. Assim, a análise de tendência na série de vazão máxima anual foi realizada utilizando os testes de Mann-Kendall e Spearman rho, a fim de verificar a significância da tendência. Os resultados dos testes são apresentados na Tabela 2. Como o nível de significância padrão adotado de $5 \%$ nos testes de tendência, tanto Mann-Kendall quanto Spearman rho, superam o valor $Z_{\alpha / 2}(1,96)$, a hipótese nula de nenhuma tendência foi rejeitada, com tendência crescente identificada, logo, a série de vazão máxima anual do Rio Pardo, não pode ser caracterizada como estacionária.

Tabela 2: Resultados da análise de tendência de vazão máxima anual do Rio Pardo na estação Clube de Regatas.

\begin{tabular}{c|c|c}
\hline Teste de tendência & Resultado & Tendência \\
\hline Mann - Kendall & 2,91 & Crescente \\
Spearman rho & 2,87 & Crescente \\
\hline
\end{tabular}

\subsubsection{Identificação do ponto de mudança}

Após a identificação da tendência na série de vazão máxima, realizou-se o teste de Pettitt para identificar em que ano ocorreu a ruptura (ponto de mudança) nos dados de vazão máxima da série histórica, marcando a presença de dois períodos hidrológicos distintos. Os resultados mostram que o ponto de mudança ocorreu no ano de 1975 (Figura 3). 


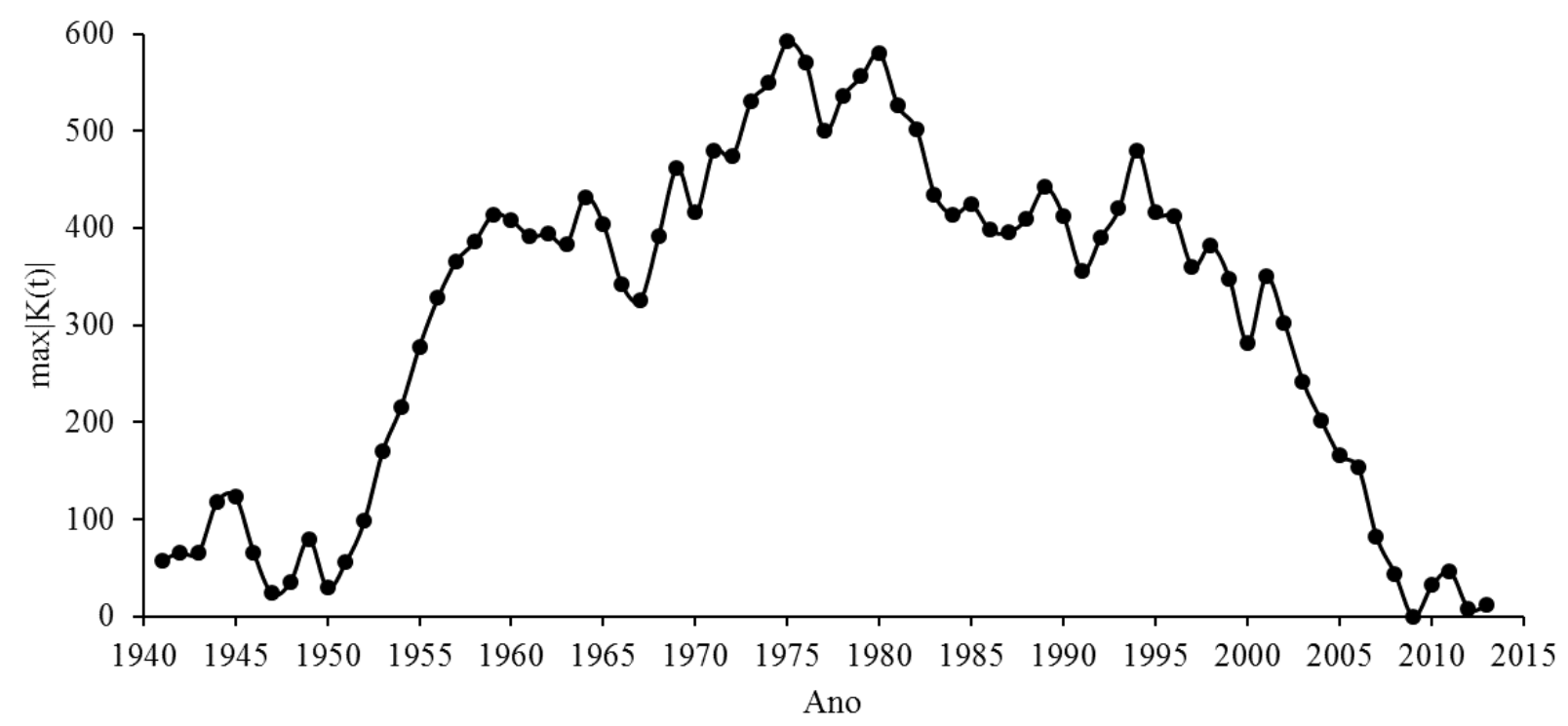

Figura 3: Ponto de mudança na série de vazão anual do Rio Pardo. Fonte: Adaptado da ANA (2016).

A partir da definição do ponto de mudança, separou-se a série de vazão máxima em dois períodos distintos, um de 1941 a 1975 e outro de 1976 a 2013 (Figura 4).

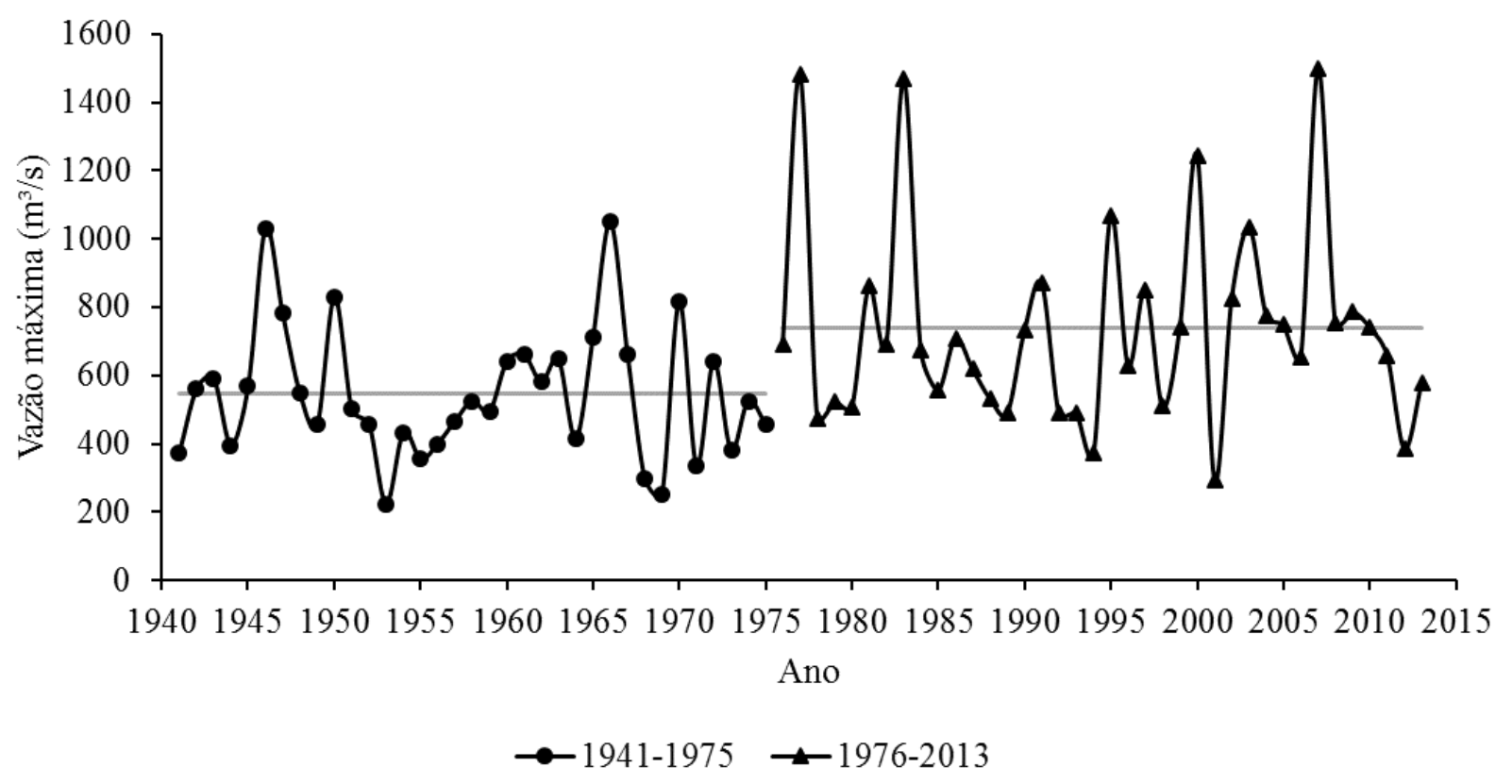

Figura 4: Mudança dos níveis de vazão máxima anual do Rio Pardo. Fonte: Adaptado da ANA (2016).

Podemos observar que após esta separação a média da vazão máxima no primeiro período foi de $545 \mathrm{~m}^{3} / \mathrm{s}$ e no segundo período apresentou média de $737 \mathrm{~m}^{3} / \mathrm{s}$, aumentando aproximadamente $26 \%$ a média da vazão máxima anual.

Com base nos dados estatísticos, é possível apontar que a série de vazão máxima anual diária no Rio Pardo não é estacionária, apresentando tendência de crescimento e que a mudança na série aconteceu por volta de 1975. Logo, segundo os vários estudos citados anteriormente, há a necessidade de investigar se a principal causa da não estacionariedade da série é devido a alterações no regime pluviométrico ou se há relação com o uso do solo. 


\subsection{Possíveis causas da não estacionariedade da vazão máxima anual}

\subsubsection{Regime pluviométrico}

Utilizando a média das 10 estações pluviométricas da região da bacia hidrográfica do Rio Pardo, aplicou-se os testes de tendência nos meses de verão austral. Constatou-se, para o nível de significância de 5\%, que a hipótese nula foi aceita, ou seja, não há tendência significativa de aumento ou redução (Tabela 3 ) na precipitação durante o período mais chuvoso da região, assim como identificado por Salviano et al. (2016) ao aplicar o teste de Mann-Kendall sobre os dados de precipitação para o Brasil.

Tabela 3: Resultados da análise de tendência da precipitação nos meses de verão austral.

\begin{tabular}{c|c|c|c}
\hline Mês & Mann - Kendall & Spearman rho & Tendência \\
\hline Dez & 1,20 & 1,33 & Nula \\
Jan & 1,71 & 1,79 & Nula \\
Fev & 0,13 & 0,10 & Nula \\
\hline
\end{tabular}

Logo os de precipitação, durante os meses de maior contribuição para a ocorrência de vazão máxima, não apresentam significância estatística para que as vazões no Rio Pardo apresentem tendência de crescimento. Estudos realizados na China por Zhang et al. (2011), apontam que em algumas bacias hidrográficas podem existir pontos de alteração estatisticamente significativa na vazão, mas não em precipitação.

\subsubsection{Uso do solo}

Um dos fatores que podem levar a não estacionariedade de uma série de vazão é o uso da terra e as mudanças na cobertura vegetal. Segundo Pitman (1978), a maioria dos registros de vazão são afetados em algum grau pelas mudanças nas atividades do homem na bacia. Utilizando os dados de Dias et al. (2016) para verificar o nível de antropização na bacia hidrográfica do Rio Pardo (apenas pastagens plantadas), podemos observar o crescimento das áreas antropizadas na bacia (Figura 5).

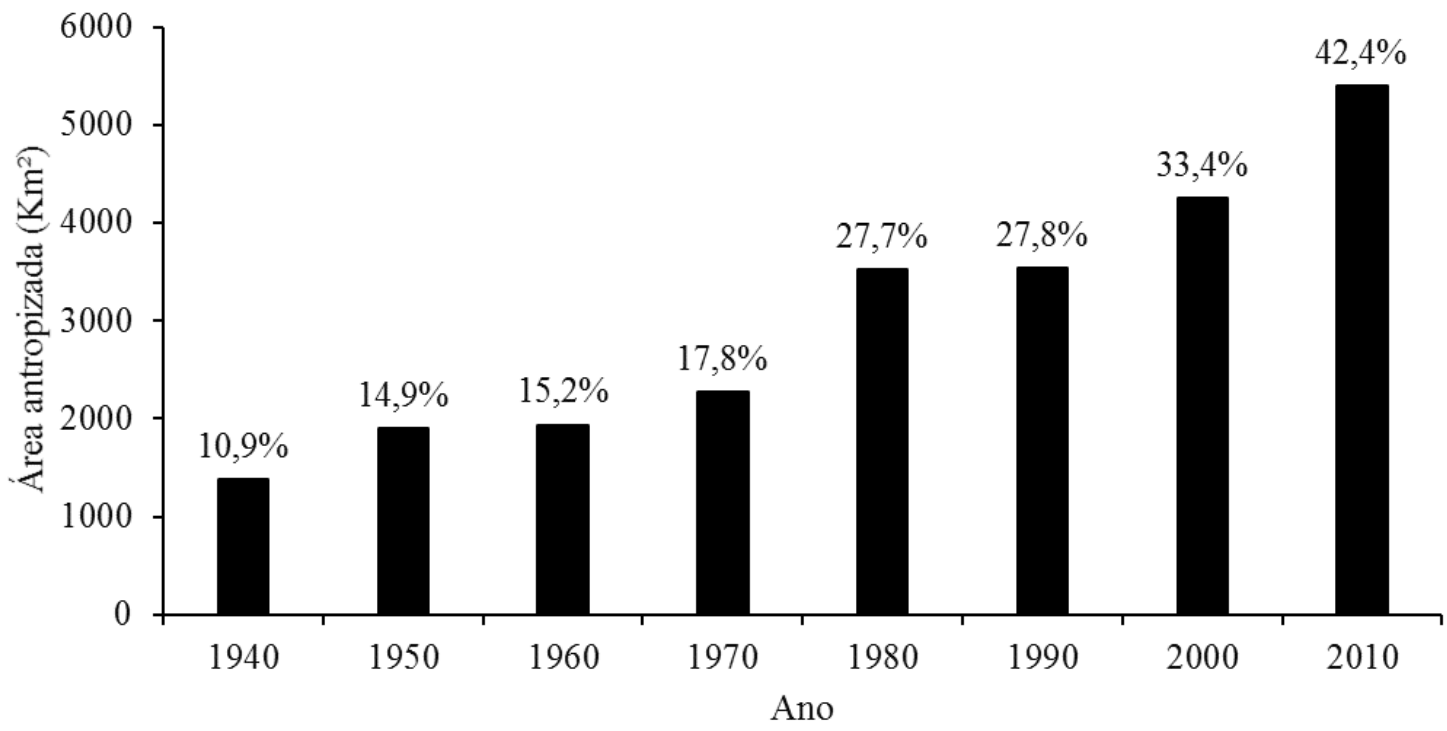

Figura 5: Precipitação máxima mensal acumulada na bacia hidrográfica do Rio Pardo. Fonte: Adaptado de Dias et al. (2016). 
Através da Figura 5, nota-se que entre os anos de 1940 a 1970, a área antropizada, através de pastagem plantada, foi de $10,9 \%\left(1385,8 \mathrm{~km}^{2}\right)$ para $17,8 \%\left(2268,4 \mathrm{~km}^{2}\right)$, ou seja, um aumento de 6,9\% $\left(882,6 \mathrm{~km}^{2}\right)$, que é pouco se comparado ao período de 1980 a 2010, tendo um salto de $27,7 \%\left(3521,0 \mathrm{~km}^{2}\right)$ para 42,4\% $\left(5395,1 \mathrm{~km}^{2}\right)$, aumentando em 14,7\% $\left(1874,1 \mathrm{~km}^{2}\right)$ durante este período. Ainda é possível observar que de 1970 a 1980 houve o aumento de 9,8\% (1252,6 km²), um valor bastante acentuado na área antropizada.

O período de 1970 a 1980, coincide justamente com a época de ruptura nos dados de vazão máxima (1975), mostrado anteriormente (Figura 03) através do teste de Pettitt. Estudos apontam que a magnitude do aumento na vazão anual é dependente da extensão da área alterada vegetal (BOSCH e HEWLETT, 1982; SAHIN e HALL, 1996) e independem da área da bacia (COE et al., 2011).

Com o auxílio da Figura 06, é possível acompanhar a evolução das áreas antropizada na bacia hidrográfica do Rio Pardo.
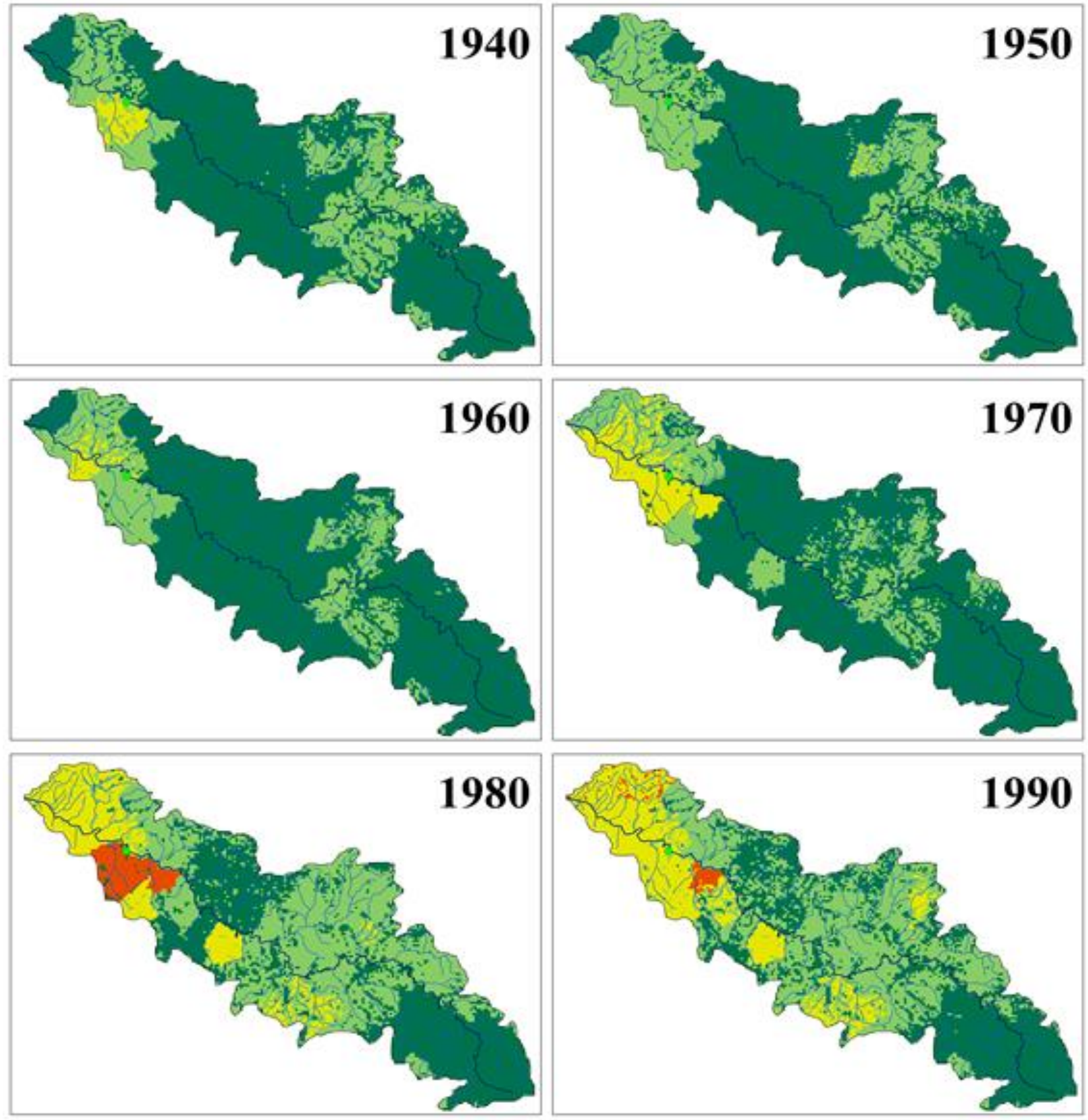


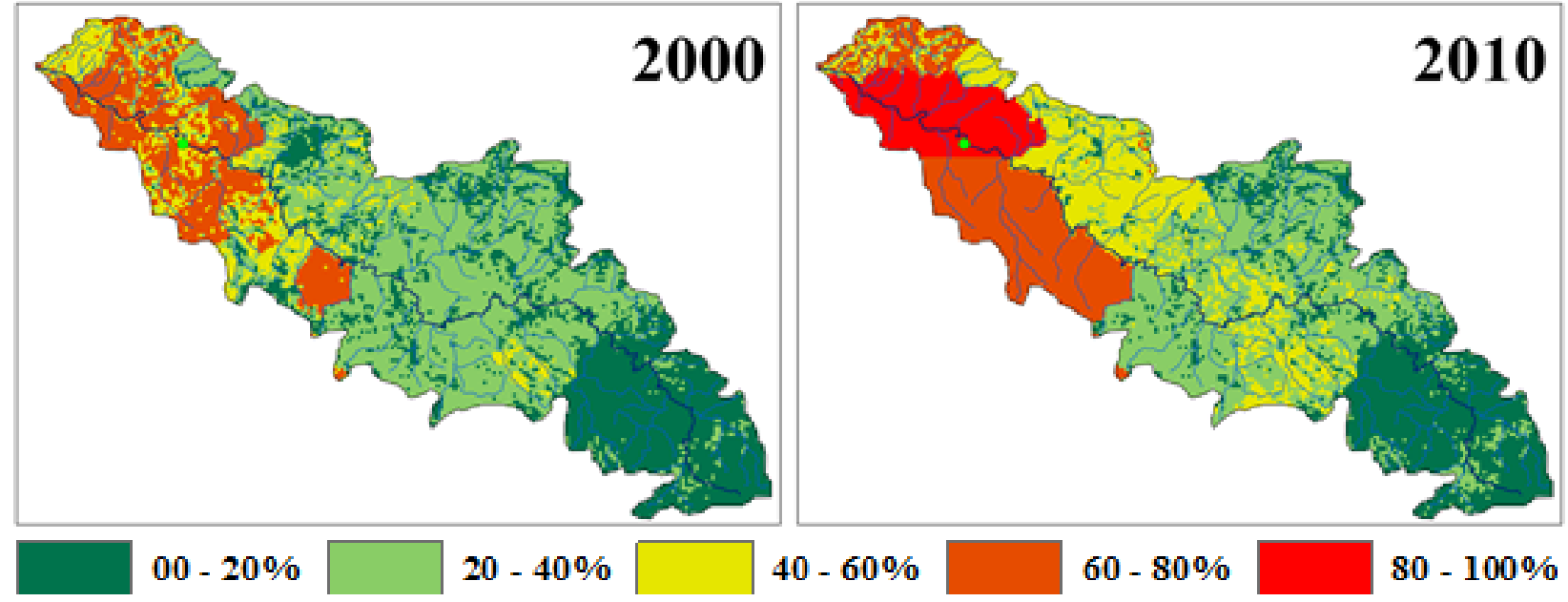

Figura 6: Evolução da área antropizada através de pastagens plantadas. Fonte: Adaptado de Dias et al. (2016).

Ao observar a Figura 6, nota-se que a antropização na bacia ocorreu em regiões estratégicas, onde a substituição da vegetação natural por pastagem plantada ou culturas agrícolas como a cana-de-açúcar (CELARINO, 2015), ocorreu de maneira mais acentuada.

Realizando uma análise conjunta da área antropizada com a elevação topográfica da bacia (Figura 1) é possível inferir que na região de maior elevação (cabeceira), ocorreu a menor alteração no uso do solo, possivelmente por ser uma região menos propícia a atividades agrícolas. Nas regiões mais planas, houve intensa alteração do uso do solo nos últimos anos, além de ser uma região próxima de grandes centros urbanos como Ribeirão Preto - SP. Segundo He et al. (2013) mudanças no uso do solo devido ao rápido desenvolvimento social e econômico tem impacto significativo sobre a resposta hidrológica, contribuindo para a variabilidade dos recursos hídricos na região.

Essa alteração no uso do solo, além de contribui para a diminuição do tempo de concentração da água precipitada na bacia, reduz o atrito e outras abstrações iniciais favorecendo o aumento das vazões máximas. De acordo com Pruski et al. (2004), a cobertura e os tipos de uso do solo, exercem influência na interceptação da água da chuva, já que quanto maior a porcentagem de cobertura vegetal e rugosidade da superfície do solo, menor será o escoamento superficial. Como observado até o ano de 2010, as regiões mais planas da bacia têm aproximadamente, de 60 a $80 \%$ de sua cobertura ocupada por pastagens plantadas, o que diminui a rugosidade da superfície e consequentemente afeta no valor da vazão máxima. Logo com a ocorrência de eventos extremos precipitação, o nível de vazão máxima pode provocar prejuízos e desastres à população.

\section{CONSIDERAÇÕES FINAIS}

De acordo com os testes estatísticos foi possível observar que a série de vazão máxima no Rio Pardo na estação fluviométrica do Clube de Regatas, não é estacionária, pois apresentou tendência de crescimento. Tais tendências iniciaram aproximadamente, a partir do ano de 1975. Como os eventos de precipitação estão estatisticamente estacionários, a substituição da vegetação natural por pastagens plantadas, representa um dos principais fatores do comportamento da série 
de vazão máxima não ser mais estacionária, contribuindo com aumento de aproximadamente $26 \%$ nas vazões máximas. Entretanto são necessários mais estudos para poder subsidiar tal hipótese.

\section{REFERÊNCIAS BIBLIOGRÁFICAS}

1. ADNAN, N. A.; ATKINSON, P. M. Exploring the impact of climate and land use changes on streamflow trends in a monsoon catchment. International Journal of Climatology, v. 31, n. 6, p. 815-831, 2011.

2. AHMAD, I.; TANG, D.; WANG, WANG, T.; WANG, M.; WAGAN, B. Precipitation Trends over Time Using Mann-Kendall and Spearman's rho Tests in Swat River Basin, Pakistan. Advances in Meteorology, vol. 2015, p. 15, 2015.

3. BAYER, D. M. Efeitos das mudanças de uso da terra no regime hidrológico de bacias de grande escala. $156 \mathrm{f}$. Tese (Doutorado em Recursos Hídricos e Saneamento Ambiental) - Universidade Federal do Rio Grande do Sul, Porto Alegre, 2014.

4. BOSCH, J. M.; HEWLETT, J. D. A review of catchment experiments to determine the effect of vegetation changes on water yield and evapotranspiration. Journal of Hydrology, v. 55, p. 323, 1982.

5. CELARINO, A. L. S. O quaternário tardio na bacia do Rio Pardo (MG e SP): análises morfométricas e evidências paleoambientais preservadas em solos e sedimentos de planícies fluviais. 2015. 220 f. Tese (Doutorado em Geografia) - Instituto de Geociências, Universidade Estadual de Campinas, Campinas - SP, 2015.

6. COE, M. T. et al. The effects of deforestation and climate variability on the streamflow of the Araguaia River, Brazil. Biogeochemistry, v.105, p. 119-131, 2011.

7. COULIBALY, P.; BALDWIN, C. K. Nonstationary hydrological time series forecasting using nonlinear dynamic methods. Journal of Hydrology, v. 307, n. 1-4, p. 164-174, 2005.

8. DALE, J.; ZOU, C. B.; ANDREWS, W. J.; LONG, J. M.; LIANG, Y.; QIAO, L. Climate, water use, and land surface transformation in an irrigation intensive watershed-Streamflow responses from 1950 through 2010. Agricultural Water Management. v. 160, p. 144-152, 2015.

9. DAMÁZIO, J. M.; COSTA, F. S. Stationarity of annual maximum daily streamflow time series in South-East brazilian rivers. Cadernos do IME. Série Estatística, v. 37, p. 29-30, 2014.

10. DETZEL, D. H. M.; MINE, M. R. M. Trends in hydrological series: methods and application. 11th International Conference on Hydroscience \& Engineering, Hamburg, Germany, 2014.

11. DIAS, L. C. P.; PIMENTA, F. M.; SANTOS, A. B.; COSTA, M. H.; LADLE, R. J. Patterns of land use, extensification, and intensification of Brazilian agriculture. Glob Change Biol. v. 22, n. 5, p. 1365-2486-16, 2016.

12. DIERMANSE, F., KWADIJK, J. C. J., BECKERS, J. V. L., AND CREBAS, J. I. Statistical trend analysis of annual maximum discharges of the Rhine and Meuse rivers, BHS third international symposium, New Castle, 2010.

13. GEDNEY, G.; COX, P. M.; BETTS, R. A.; BOUCHER, O.; HUNTINGFORD, C.; STOTT, P. A. Detection of a direct carbon dioxide effect in continental river runoff records. Nature, v.439, p. 835-837, 2006.

14. GAN M. A., KOUSKY V. E., ROPELEWSKI C.F. The South America Monsoon Circulation and Its 
Relationship to Rainfall over West-Central Brazil. Journal of Climate. v.17(1): 47-66, 2004.

15. HE, Y.; LIN, KAIRONG; CHEN, X. Effect of Land Use and Climate Change on Runoff in the Dongjiang Basin of South China. Mathematical Problems in Engineering, v. 2013, p. 14, 2013.

16. KAHYA, E.; KALAYCI, S. Trend analysis of streamflow in Turkey. Journal of Hydrology. 289: 128144, 2004.

17. KENDALL, M. G. Rank correlation measures. Charles Griffin: London, U.K, p. 220, 1975.

18. KIBRIA, K. N.; AHIABLAME, L.; HAY, C.; DJIRA, G. Streamflow Trends and Responses to Climate Variability and Land Cover Change in South Dakota. Hydrology, v.3 n. 1, 2016.

19. KOCHENDORFER, J. P.; HUBBART, J. A. The roles of precipitation increases and rural land-use changes in streamflow trends in the upper Mississippi river basin. Earth Interact. v.14, p.1-12, 2010.

20. LABAT, D.; GODDERIS, Y.; PROBST, J. L.; GUYOT, J. L. Evidence for global runoff increase related to climate warming. Adv. Water Resour. v.27, p. 631-642, 2004.

21. LEHMANN, E. L. Nonparametrics, Statistical Methods Based on Ranks, Holden-Day, San Francisco, California, USA, 1975.

22. MANN, H. B. Non-parametric test against trend. Econometrika, v. 13, n. 3, p. 245-259, 1945.

23. MILLY, P. C. D.; DUNNE, K. A.; VECCHIA, A. V. Global pattern of trends in streamflow and water availability in a changing climate. Nature, v.438, p. 347-350, 2005.

24. MILLY, P. C. D.; BETANCOURT, J.; FALKENMARK, M.; HIRSCH, R. M.; KUNDZEWICZ, Z. W.; LETTENMAIER, D. P.; STOUFFER, R. J. Stationarity is dead: whither water management? Science, 319, 573-574, 2008.

25. PENEREIRO, J. C.; FERREIRA, D. H. L. Testes estatísticos e questões ambientais no ensino de engenharia: uma aplicação em climatologia. Revista de Ensino de Engenharia, v. 31, p. 25-44, 2012.

26. PETTITT, A. N. A non-parametric approach to change point problem. Appl. Stat., London v. 28, n. 2 p.126-135, 1979.

27. PITMAN, W. V. Trends in streamflow due to upstream land use changes. J. Hydrol., 39: 227237, 1978.

28. PRUSKI, F. F.; SILVA, D. D.; BRANDÃO, V. S. Escoamento Superficial. Viçosa: UFV. 2. ed. 2004.

29. QUEIROZ, M. A. Avaliação de tendências em séries de precipitação diária máxima anual na faixa central do estado de Minas Gerais. 2013. 202 f. Dissertação (Mestrado em Saneamento, Meio Ambiente e Recursos Hídricos) - Escola de Engenharia, Universidade Federal de Minas Gerais, Belo Horizonte - MG, 2013.

30. SALVADORI, N. Evaluation of non-stationarity in annual maximum flood series of moderately impaired watersheds in the upper Midwest and Northeastern United States. Dissertation (Master of Science in Environmental Engineering) - Department of Civil and Environmental Engineering, Michigan Technological University, Michigan, 2013.

31. SAHIN, V.; HALL, M. J. The effects of afforestation and deforestation on water yields. Journal of Hydrology, v.178, p.193-309, 1996.

32. SALVIANO, M. F.; GROPPO, J. D.; PELLEgRINO, G. Q. Análise de Tendências em Dados de 
Precipitação e Temperatura no Brasil. Revista Brasileira de Meteorologia, v. 31, n. 1, p. 64-73, 2016.

33. SANCHES, F. O.; VERDUM, R.; FISCH, G. Estudo de tendência de chuvas de longo prazo. Rev. Ambient. Água, v. 8, n. 3, p. 214-228, 2013.

34. SHADMANI, M.; MAROFI, S.; ROKNIAN, M. Trend analysis in reference evapotranspiration using Mann-Kendall and Spearman's Rho tests in arid regions of Iran. Water Resour. Manag., v. 26, n. 1, p. 211-224, 2012.

35. SHARKEY, P.; KILLICK, R. Nonparametric Methods for Online Changepoint Detection. Lancaster Univ, Lancaster, UK. 2014.

36. SNEYERS, R. On the statistical analysis of series of observations, Technical Note 143, WMO no. 415, World Meteorological Organization, 1990.

37. TAN, X. Z.; GAN, T. Y. Nonstationary analysis of annual maximum streamflow of Canada, Journal of Climate, 28 (5), 2015.

38. TUCCI, C. E. M. Impactos da variabilidade climática e uso do solo sobre os recursos hídricos. Brasília: Fórum Brasileiro de Mudanças Climáticas, p. 150, 2002.

39. ULIANA, E. M.; DEMETRIUS, D. S.; ULIANA, E. M; RODRIGUES, B. S.; CORRÊDO, L. P. Análise de tendência em séries históricas de vazão e precipitação: uso de teste estatístico não paramétrico. Revista Ambiente \& Água, v. 10, p. 82-88, 2015.

40. VEZZOLI, R.; PECORA, S.; ZENONI, E. Data analysis to detect inhomogeneity, change points, trends in observations: an application to Po river discharge extremes. Research Papers Issue RP0138 Technical report. CMCC; 2012.

41. VILANOVA, M. R. N. Trends in Mean Annual Streamflows in Serra da Mantiqueira Environmental Protection Area. Braz. arch. biol. technol. v. 57, n. 6, p. 1004-1112, 2014.

42. WANG, W.; VAN GELDER, P. H. A. J. M.; VRIJLING, J. K. Trend and stationarity analysis for stareamflow processes of rivers in Western Europe in the 20th century, IWA International Conference on Water Economics, Statistics, and Finance, Rethymno, Greece, 8-10 July 2005.

43. XIONG, L.; GUO, S. Trend test and change-point detection for the annual discharge series of the Yangtze River at the Yichang hydrological station. Hydrological Sciences Journal, v. 49, n. 1, p. 99-112, 2004.

44. YEH, C.; WANG, J.; YEH, H.; LEE, C. Spatial and Temporal Streamflow Trends in Northern Taiwan. Water, v.7, n.2, p. 634-651, 2015.

45. YUE S.; PILON P.; CAVADIAS G. Power of the Mann-Kendall and Spearman's rho tests for detecting monotonic trends in hydrological series. Journal of Hydrology, v. 259, n. 1-4 p. 254271, 2002.

46. ZHANG, Z.; CHEN, X.; XU, C-Y.; YUAN, L.; YONG, B.; YAN, S. Evaluating the non-stationary relationship between precipitation and streamflow in nine major basins of China during the past 50 years. Journal of Hydrology, v. 409, n. 1-2, p. 81-93, 2011.

47. ZHANG, Y.; CAI, W.; CHEN, Q.; YAO, Y.; LIU, K. Analysis of Changes in Precipitation and Drought in Aksu River Basin, Northwest China. Advances in Meteorology, v. 2015, p. 15, 2015. 